\title{
Collaborative Learning in Heterogeneous Classes Towards a Group Formation Methodology
}

\author{
Razmerita, Liana; Brun, Armelle
}

Document Version

Final published version

Published in:

Proceedings of the 3rd International Conference on Computer Supported Education

Publication date:

2011

\section{License \\ CC BY-NC-ND}

Citation for published version (APA):

Razmerita, L., \& Brun, A. (2011). Collaborative Learning in Heterogeneous Classes: Towards a Group

Formation Methodology. In Proceedings of the 3rd International Conference on Computer Supported Education (pp. 189-194)

Link to publication in CBS Research Portal

\section{General rights}

Copyright and moral rights for the publications made accessible in the public portal are retained by the authors and/or other copyright owners and it is a condition of accessing publications that users recognise and abide by the legal requirements associated with these rights.

Take down policy

If you believe that this document breaches copyright please contact us (research.lib@cbs.dk) providing details, and we will remove access to the work immediately and investigate your claim. 


\title{
COLLABORATIVE LEARNING IN HETEROGENEOUS CLASSES Towards a Group Formation Methodology
}

\author{
Liana Razmerita ${ }^{1}$ and Armelle Brun ${ }^{2}$ \\ ${ }^{1}$ Copenhagen Business School, CBS, ISV, Dalgas Have, 15, DK-2000 Frederiksberg, Denmark \\ ${ }^{2}$ LORIA - Nancy Université, Campus Scientifique, 54506 Vandoeuvre les Nancy, France \\ lr.isv@cbs.dk,armelle.brun@loria.fr
}

\begin{abstract}
Keywords: Group formation, Group work, Collaborative learning, Web 2.0.
Abstract: $\quad$ Group work has been adopted as an important tool to support collaborative work in order to enhance learning processes. There is a wealth of literature related to group performance and the impact of group composition on group and individual performance. However, very few studies address the issue on how to automatically form groups. This article proposes a methodology that could be used by professors to form groups automatically taking into account different criteria as well as the students' profile. This methodology is based on a pilot study that analyzes group composition of self-formed student groups. The pilot study findings suggest that students tend to form homogeneous group in terms of level of the knowledge. Furthermore, students report that working on common topics of interests was a decisive factor in forming the groups.
\end{abstract}

\section{INTRODUCTION}

Group work and collaborative work are important pedagogical tools for classroom assignments and have proved to have a strong impact on individual and cooperative learning (Johnson and Johnson, 1994). Furthermore Web2.0 has created new possibilities for students to engage, interact and collaborate in various learning tasks that may enhance learning processes and the overall learning experience. In this context the didactic challenge for educators is to design and integrate a new set of tools based on specific didactic principles associated with a specific domain of learning (Mondahl et al., 2009). Consider a professor who wants to assign group work to students during a course. One issue he/she has to handle is: How to form groups? He/she may either let the students form the groups by themselves or may form student groups randomly or based on their physical proximity (e.g. their position in the class). Even though this is a very easy convenient approach, randomly formed groups may not be fair or may not be the best approach. Group work has many variables and different factors that can influence group performance. For example, literature related to motivation and group learning shows that performance is not only linked to the interest in the subject to be learned but may also be related to relations to peers, gender differences, age, individual differences, cultural backgrounds within the gro- up, personality traits, etc. (Souren et al., 2003). Furthermore, explaining to students why they form a group and why they are performing certain tasks may lead to better performance (Bekele, 2005). Moreover, a student has to feel comfortable in a group in order to communicate his/her ideas, to express his point of view with his/her group-mates. Thus, a professor may select a special criterion based on which he/she wants to create the groups. This criterion can be either a single criterion or a set of different criteria (e.g. heterogeneous in relation with their background). However forming groups of students, especially for large size classes, is an intractable and time consuming task for the professor.

Previous studies related to collaboration and group work have emphasized the importance of heterogeneity for performance, creativity and learning (Rich, 1997). A heterogeneous group is made of members that are balanced in terms of diversity based on some criteria: culture, gender, personality, etc.). There is a wealth of literature related to group performance and their composition (Slavin, 1995; Sharan, 1999) but very few literature addresses the issue of how to automatically form groups. Group work and collaborative learning open a number of challenging research questions related to group performance and group composition such as: How heterogeneity or homogeneity of a group influence the group performance and students' learning? Do homogenous 
groups perform better than heterogeneous groups? How students form groups? Literature suggests that groups should be formed differently according to the type of assignments. Homogeneous groups are better for achieving specific goals (e.g. short-term and guided problem solving) while heterogeneous groups are better for long-term knowledge discovery problems (Bekele, 2005).

This article is a preliminary study that focuses on how students form groups and proposes a method to form groups automatically, taking into account different criteria as well as the students' profile. This paper relies on a preliminary study case investigating the way students form groups, in the framework of a course where they have to conduct research and present project related ideas and findings in group.

The article is structured in five sections. The second section presents an overview of literature on collaborative learning, collaborative work, group composition and group formation. The third section presents a pilot study of group work within a heterogeneous classroom supported by a Web 2.0-enabled learning environment. The fourth section proposes a methodology that enables professors in very different teaching areas to form groups automatically.

\section{LITERATURE REVIEW}

\subsection{Web 2.0 and e-Learning 2.0}

Web 2.0 and the associated technologies have changed the way the web is used; web is a dynamic social space where participation, collaboration, online interaction are core elements. Web 2.0 or social software may be approached from different perspectives: as a new social media tool, a facilitator of new forms of interaction and knowledge sharing (Kirchner et al., 2008), an enabler of personal information and knowledge management tools (Razmerita and Kirchner, 2009) and new didactic tools that facilitate interaction and social processes. Web 2.0 has a large influence on learning approaches (e.g. using wikis, blogs, micro blogs) and it offers new means to interact, socialize on-line, find information, and communicate using a wide range of new collaborative services. Learning is not anymore viewed as a unidirectional process, where teachers are in the same place at the same moment with the learners, and knowledge is transferred from teachers to learners. Learners are now participants in the learning process thanks to the tools that enable and encourage them to participate, interact and collaborate more easily with other learners, teachers or peers, etc.

\subsection{Collaborative Learning and Cooperative Learning}

Collaborative learning can be described as a situation in which two or more people learn or attempt to learn something together. Collaborative learning is different from cooperative learning. In cooperative learning peers split the work in tasks and tend to solve these tasks individually and then assemble their results into the final output. While in collaborative learning learners interact and do work together in order to complete their assignments. Despite the difference, many articles use these two terms interchangeable. For example, cooperative learning (CL) is defined as an instructional strategy in which students work actively and purposefully together in small groups to enhance both their own and their teammates' learning (Abrami et al., 2004). Literature emphasizes that collaborative learning is one of the most successful techniques to enhance student performance. Several studies report that group work and cooperative learning enhance the learning of an individual compared to when he/she learns alone (Dansereau and Johnson, 1994; Slavin, 1983). Several approaches have been proposed to support collaborative learning with the aim of facilitating information and resource sharing between students (Florea, 1999; Krejins et al., 2002). Group work can be performed either by students physically present at the same place and at the same time (synchronous work) or remotely through asynchronous work (Souren et al., 2003).

\subsection{Group Composition}

The quality of the learning process in the context of collaborative work highly depends on the characteristics of the group. Previous studies suggest that groups should be formed randomly or by students themselves and groups should have a small size, made up to four-members of different level of knowledge (Slavin, 1987). Related work emphasized the importance of personality attributes, gender, school background, ethnic background, motivation (Bradley and Herbert, 1997; Alfonseca et al., 2006) in group performance. The learning style is also an important criterion in group composition (Martin and Paredes, 2004; Wang et al., 2007). It has been observed that the quality of learning in groups is influenced by their diversity. Heterogeneous groups may outperform homogeneous groups (Nijstad and Carsten, 2002). Some studies emphasized that heterogeneous groups may be more creative and innovative (Paulus and Nijstad, 2003) and furthermore they may be more effective for individual learning. One of the first het- 
erogeneity criteria was in relation with the level of knowledge and skills (Slavin, 1987; Webb, 1989). Diversity in background, opinions (Siemens, 2010), ideas, personality, gender, are also criteria that can be considered in the heterogeneity (Slavin, 1995). Groups may be automatically formed based on thinking styles (Wang et al., 2007), competence, learning style and interactions (Ho et al., 2009), learning achievements (Chan et al., 2010). The clustering approaches proposed in the literature use genetic algorithms (Graf and Bekele, 2006; Ho et al., 2009; Chan et al., 2010) or ant colony optimization (Bekele, 2005; Graf and Bekele, 2006).

\section{THE PILOT STUDY}

\subsection{The StudyBook Context}

This pilot study aims to investigate the use of a Web 2.0 collaborative platform, StudyBook, to support new ways of teaching and learning. The underlying hypothesis of the overall study is that collaborative groupwork creates natural opportunities for the learners to articulate their understanding, reflect on and justify actions and these activities may improve learning. Furthermore new Web2.0/Web3.0 technologies integrated in a learning platform may support group work, collaboration and learning processes in new ways that are more natural for new generation of learners. In particular, the StudyBook project aims to investigate the use of social-collaborative services for collaborative learning and groupwork (Mondahl et al., 2009). A screenshot of StudyBook is presented in the Figure 1. This study has been conducted within the Web Interaction Design and Communication course, an elective course for bachelor students at the Copenhagen Business School (CBS) in Denmark. This course enrolled 46 bachelor students from seventeen different countries and from different study programs (Business Administration, European studies, International Business, Marketing, HRM, etc). Within this course, a project has to be conducted collaboratively, partly in group and partly individually. The students had to select a topic of interest, to form groups and work in group on this topic. Within their projects, the students define their own topic to work on in relation with their core areas of interest and the main course topics. The project work is divided in two steps. 1)Work in group: The group has to work on a common topic of interest. The StudyBook platform facilitates students' interaction and group collaboration. Students are not in the same study programs and do not have common free time-slots, their

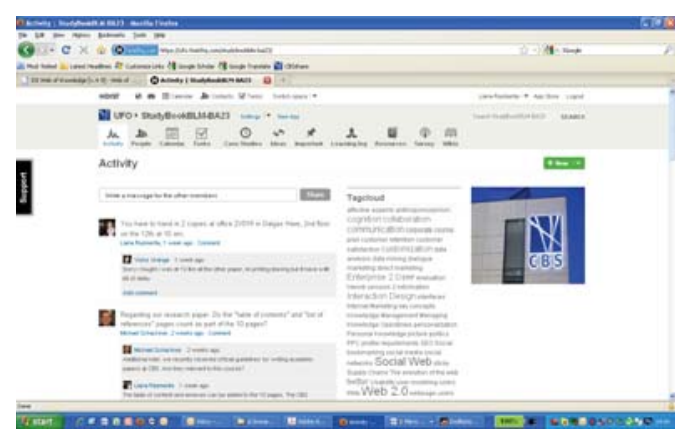

Figure 1: Screenshot of the StudyBook platform.

project work had to be constructed collaboratively using wikis and afterwards had to make a presentation. 2) Work individually: In the second step, students have to continue to work on the selected topic individually in order to write a ten page report that will be graded.

\subsection{Data Collection: The Questionnaire}

Two questionnaires have been designed with two objectives in mind: to assess the student's perception of StudyBook as a learning platform and collect data about students and the way they form groups. Within the following section of the article we focus on the findings related to the analysis of the groups that have been formed. In the questionnaire, data about students include their background (study programs), culture (the country they come from), topics of interests and level of knowledge in relation with the course.

Students had to answer about both their level of knowledge (beginner, intermediate, advanced) and research interests (not interested, interested, very interested). In addition, students were asked about the way they have formed their groups.

\subsection{Data Analysis: Evaluation of Groups}

This section presents an analysis about the way groups have been formed in the heterogeneous classe. Among the 46 students enrolled in the course, 29 students have answered the questionnaire. Eight groups in total have answered the questionnaire. First of all, students declare they have enjoyed working in groups and have perceived positively their workgroup collaboration. A first analysis of the student provided answers shows that the students who answered the questionnaire are from fifteen different countries all over the world. The most represented countries are Denmark, Spain and France (4 students from each coun- 
Table 1: Average distances within groups.

\begin{tabular}{|l|c|c|}
\hline Group & level of knowledge & topics of interest \\
\hline Group1 & 0.02 & 0.27 \\
Group2 & 0.48 & 0.10 \\
Group3 & 0.24 & 0.23 \\
Group4 & 0.16 & 0.15 \\
Group5 & 0.22 & 0.20 \\
Group6 & 0.02 & 0.05 \\
Group7 & 0.05 & 0.18 \\
Group8 & 0.24 & 0.22 \\
All Gr & 0.18 & 0.18 \\
Class & 0.23 & 0.18 \\
\hline
\end{tabular}

try) and Hong Kong (3 students). Thus, the formed groups are probably heterogeneous in terms of cultural background. In our overall study the heterogeneity is related to students' background (different study programs), cultural (different countries), topics of interests and the level of knowledge. In this preliminary study, we specifically focus on level of knowledge and topics of interest. In order to study homogeneity and heterogeneity of groups we compute a metric distance between students taking into account their declared level of knowledge or/and topics of interests.

\subsubsection{Group Heterogeneity in Relation with the Level of Knowledge}

Studies suggest that if students organize themselves in groups, they usually tend to form homogeneous groups (Souren et al., 2003). The first column of Table 1 represents the average distance in terms of level of knowledge within groups and within the class. The average distance between all students in the class is 0.23 and standard deviation is 0.17 . Less than $3 \%$ of the pairs of students have a distance greater than 0.8 . This means that few students are very different in terms of their declared level of knowledge. Among the eight groups, the average distance is 0.18 , the most homogeneous group has an average distance of 0.02 and the less homogeneous has an average distance of 0.48 . Three groups have an average distance less than 0.05 . The average distance between students in the groups is smaller than the average distance in the whole matrix. Thus groups formed by students tend to be similar in terms of knowledge. The findings of our study confirm the fact that students tend to form homogeneous groups.

\subsubsection{Group Heterogeneity in Relation with their Topics of Interests}

The second column of Table 1 represents the average distance in terms of topics of interest within groups and within the class. The average distance between all students in the class is 0.18 and standard deviation is 0.12 . Less than $0.2 \%$ have a distance greater than 0.7. Despite their heterogeneity, students appear to be quite homogeneous in relation with their level of interest. Among the eight groups, the average distance is also 0.18 , which is similar to the average distance among groups in terms of level of knowledge. The most homogeneous group has an average distance of 0.05 and the less homogeneous one has an average distance of 0.27 . Five groups out of the eight have similar average interest distance and average knowledge distance. Within the class, the average distance between students in terms of topics of interest (0.18) is lower than the one in terms of level of knowledge (0.23); students are more similar in terms of topics of interest than in level of knowledge. However, the average distance within groups is similar to the average distance within the class; thus we can deduce that students do not tend to form similar groups in terms of topics of interest.

\subsubsection{How Students Have Formed Groups?}

Based on the assignment requirements described in section 3.1, the results of the study in relation with how students form groups are presented in Figure 2. Based on the questionnaire's answers, a large majority of students have declared forming groups based on identified common topics of interests $(68,97 \%)$, followed by affinity with the others members $(10,34 \%)$ and different backgrounds and culture (10,34\%).
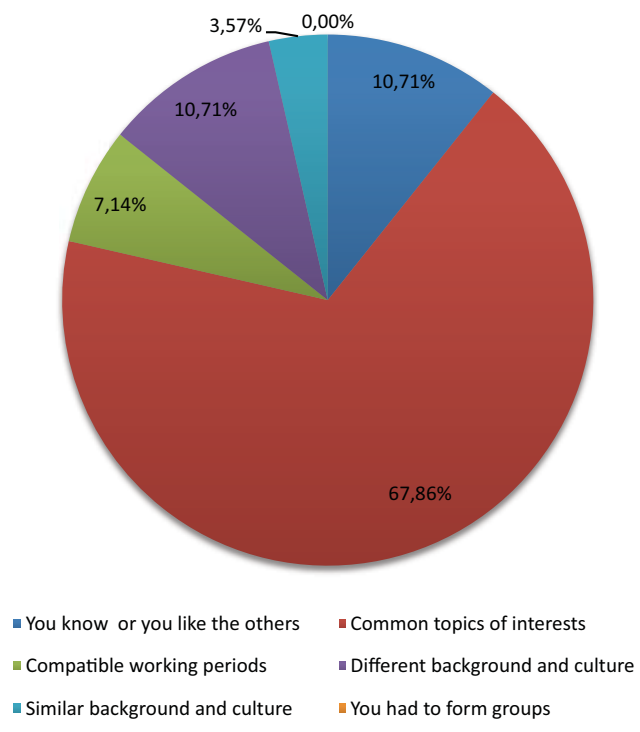

Figure 2: Reported criteria on groups formed. 
As a conclusion, the groups formed by students tend to be homogeneous in terms of the level of knowledge. However, these groups are not really homogeneous in terms of the topics of interests, whereas the students answered in the questionnaire they formed homogeneous groups in terms of common topics of interests. Thus, when leaving students to form groups by themselves, the resulting groups may not have the expected characteristics. Thus a tool that automatically forms groups may be highly useful to ensure that groups have given characteristics and lead to the expected type of learning and performance. In the following section, we propose a methodology to form groups automatically according to students' profile and specified citeria.

\section{METHODOLOGY FOR FORMING GROUPS AUTOMATICALLY: CLUSTERING STUDENTS}

The proposed methodology comprises four main steps: collecting data about students, initialize the vectors representing the students' characteristics, clustering students, evaluating the group performance.

Step 1. Collect data about students. Questionnaires provide an effective way to collect data about the students. However data can be provided by students or by the administration (e.g., the study program, the background) or by the professor (possible topic, learning concepts, learning objectives, students' skills, heterogeneous versus homogeneous groups). As described previously, in our pilot study the following data (topics of interest, level of knowledge, country of origin, study program) was collected with the purpose of automatic group formation and other purposes. The collected data needs to be preprocessed in order to be used by the algorithm. For example qualitative data needs to be transformed into quantitative data.

Step 2. Initialize the input vectors. Each student is represented by a vector with features/components that are made up of the attributes values associated with the student, initialized from the questionnaire.

Step 3. Select and run the clustering algorithm (e.g. $\mathrm{K}$-means, hierarchical clustering, etc) in order to generate the groups; depending on the algorithm select the number of clusters, the size of the cluster or the quality criterion. One distance measure that can be used to compute similarity between students in groups is for example the Euclidean distance:

$$
d(x, y)=\sum_{i}^{n}\left|x_{i}-y_{i}\right|
$$

where $x=\left[X_{i}\right] i=1 . . n$ represents one student's profile and $y=i=1 . . n$ represents another student's profile. Once the grouping task is achieved, the students can work on their assignments in groups formed.

Step 4. Evaluate the group performance in relation with the selected criterion or criteria. Depending on the assigned task and the learning objectives, the professor might decide to evaluate the performance of the groups and may decide to change or keep the type of clustering method for the following assignments. The performance may be evaluated at either group level or individual level.

\section{CONCLUSIONS}

This paper investigates group formation in the context of a collaborative learning platform. This paper proposes a methodology to form groups of students which relies on a preliminary study on how students formed groups within a course in a heterogeneous class. A pilot study has been conducted to study the way students form groups in the context of heterogeneous classes. According to the questionnaires findings, students have enjoyed working in groups and have perceived positively their workgroup collaboration. However not all students in the class have formed a group. Furthermore, the analysis of the groups reveals that students tend to form homogeneous groups in terms of level of knowledge, which is in line with what other previous studies have suggested (Souren et al., 2003). In relation with the topics of interests, according to the students' answers, the groups were formed based on topics of interests but according to the analysis of groups as presented in section 3.3.2 groups are not as homogeneous as students declared. The proposed methodology using clustering algorithm tools for group formation is a useful tool to help professors form groups automatically using a certain criteria. We plan to further test the methodology and the performance of automatically formed groups within different type of courses and assignments. In further studies we will assess the performance of automatically formed groups using different clustering methods. 


\section{REFERENCES}

Abrami, P. C., Poulsen, C., and Chambers, B. (2004). Teacher motivation to implement an educational innovation: Factors differentiating users and non-users of cooperative learning. Educational Psychology, pages 201-216.

Alfonseca, E., Carro, R., Martn, E., Ortigosa, A., and Paredes, P. (2006). The impact of learning styles on student grouping for collaborative learning: A case study. User Modeling and User-Adapted Interaction Journal, pages 377-401.

Bekele, R. (2005). Computer-Assisted Learner Group Formation Based on Personality Traits. PhD thesis, Hamburg University.

Bradley, J. H. and Herbert, F. J. (1997). The effect of personality type on team performance. Journal of Management Development, pages 337-353.

Chan, T., Chen, C. M., Wu, Y. L., Jong, B. S., Hsia, Y. T., and Lin, T. W. (2010). Applying the genetic encoded conceptual graph to grouping learning. Expert Systems with Applications, 37:4103-4118.

Dansereau, D. and Johnson, D. (1994). Learning, Remembering, Believing: Enhancing Human Performance, chapter Cooperative learning, pages 83-111. Druckman, D. and Bjork, R.A.

Florea, A. M. (1999). An agent-based collaborative learning system: Advanced research in computers and communications in education. In Proc. of the Int. Conf. on Computers in Education.

Graf, S. and Bekele, R. (2006). Forming heterogeneous groups for intelligent collaborative learning systems with ant colony optimization. In Intelligent Tutoring System Conference.

Ho, T. F., Shyu, S. J., Wang, F. H., and Li, C. T. (2009). Composing high-heterogeneous and high-interaction groups in collaborative learning with particle swarm optimization. In World Congress on Computer Science and Information Engineer-ing.

Johnson, D. W. and Johnson, R. T. (1994). Perspectives on Small group Learning: Theory and practice, chapter What is cooperative Learning? Rubicon Publishing Inc.

Kirchner, K., Razmerita, L., and Sudzina, F. (2008). Web 2.0: The Business Model, chapter New Forms of Interaction and Knowledge Sharing on Web 2.0. Springer Science and Business Media.

Krejins, K., Kirschner, P. A., and Jochems, W. (2002). The sociability of computer-supported collaborative learning environ-ments. Educational Technology and Society, pages 26-37.

Martin, E. and Paredes, P. (2004). Using learning styles for dynamic group formation in adaptive collaborative hypermedia systems. In Proceeding of the conference AHCWS

Mondahl, M., Rasmussen, J., and Razmerita, L. (2009). Web 2.0 applications, collaboration and cognitive processes in case-based foreign language learning. In $2 n d$ World Summit On The Knowledge Society.
Nijstad, B. and Carsten, K. (2002). Creativity and group innovation. Applied Psychology, 51(3).

Paulus, P. B. and Nijstad, B. A. (2003). Group Creativity: Innovation through Collaboration. Oxford University Press.

Razmerita, L. and Kirchner, K. (2009). Personal knowledge management: The role of web 2.0 tools for managing knowledge at individual and organisational levels. Online Information Review, 33(6):1021-1039.

Rich, M. (1997). A learning community on the internet: An exercise with masters students. In Proc. of Americas Conf. on Information Systems.

Sharan, S. (1999). Handbook of cooperative learning methods. Westport.

Siemens, G. (2010). Connectivism: A learning theory for digital age. http://www.elearnspace.org/ Articles/connectivism.htm retrieved the 16th of May.

Slavin, R. E. (1983). When does cooperative learning increase achievement? Psychological Bulletin.

Slavin, R. E. (1987). Developmental and motivational perspectives on cooperative learning: A reconciliation. Child Development, pages 1161-1167.

Slavin, R. E. (1995). Cooperative learning: Theory, research, and Practice. Allyn \& Bacon.

Souren, P., Seetharaman, P., Samarah, I., and Mykytyn, P. (2003). Impact of heterogeneity and collaborative conflict management style on the performance of synchronous global virtual teams. Information and Management, pages 303-321.

Wang, D. Y., Lin, S. J., and Sun, C. T. (2007). Diana: A computer-supported heterogeneous grouping system for teachers to conduct successful small learning groups. Computers in Human Behavior.

Webb, N. (1989). Peer interaction and learning in small groups. Intern. Journal of Educational Research, pages $21-39$. 